\title{
Spitzer Science operations: the good, the bad, and the ugly
}

\section{Deborah A. Levine}

Deborah A. Levine, "Spitzer Science operations: the good, the bad, and the ugly," Proc. SPIE 7016, Observatory Operations: Strategies, Processes, and Systems II, 70160C (12 July 2008); doi: 10.1117/12.789871

Event: SPIE Astronomical Telescopes + Instrumentation, 2008, Marseille, France 


\title{
Spitzer Science Operations: The Good, the Bad and the Ugly
}

\author{
Deborah. A. Levine \\ Spitzer Space Telescope Science Center (SSC), Infrared Processing and Analysis Center (IPAC), \\ MS 314-06 California Institute of Technology, 1200 E California Blvd., Pasadena, CA 91125
}

\begin{abstract}
We review the Spitzer Space Telescope Science Center operations teams and processes and their interfaces with other Project elements -- what we planned early in the development of the science center, what we had at a launch and what we have now and why. We also explore the checks and balances behind building an organizational structure that supports constructive airing of conflicts and a timely resolution that balances the inputs and provides for very efficient on-orbit operations. For example, what organizational roles are involved in reviewing observing schedules, what constituency do they represent and who has authority to approve or disapprove the schedule.
\end{abstract}

Keywords: Observatory Operations, Space-based observatories, Science Operations, Spitzer Space Telescope

\section{INTRODUCTION}

The Spitzer Space Telescope is the fourth and final element in NASA's family of Great Observatories. Spitzer carries an 85-centimeter cryogenic telescope and three cryogenically cooled science instruments capable of performing imaging and spectroscopy in the 3.6 to 160 micron range. Spitzer was launched on a Delta $7920 \mathrm{H}$ from Cape Canaveral into an Earth-trailing heliocentric orbit in August 2003. Current lifetime estimates indicate an expected cryogenic lifetime of about 5 1/2 years, until April 2009, substantially exceeding the lifetime requirement. The regime of infrared observing has been described as "the old, the cold and the dusty" as infrared wavelengths are particularly suited to studying high redshift objects, objects which are too cold to have significant flux at visible wavelengths and objects which are obscured by dust at visible wavelengths.

The Space Infrared Telescope Facility Science Center (SSC) at the California Institute of Technology is responsible for all aspects of science operations for Spitzer. SSC processes and archives all the science data, administers the time allocation process and observer funding, provides technical user support, conducts EPO and Public affairs activities, supports operation of the science instruments and plans and schedules observations on the observatory. SSC is currently building the Heritage Archive for the Spitzer data, which will ultimately be hosted by the NASA/IPAC Infrared Science Archive (IRSA).

This paper captures the opinions and recollections of the author, who was charged with "Operations Planning" for SSC, then became the "Operations Team Lead" and who, as of this writing, has subsided into the somewhat less hands-on role of "Operations Scientist". A number of more sober papers have been published outlining the technical aspects of the observatory and science operations - this is a more personal discourse on operations planning and lessons learned. As such, she has taken sole authorship to protect the innocent, but wishes to acknowledge that there is no way SSC could have achieved the outstanding performance it has without an extremely gifted and dedicated team, all of whom, as in Lake Woebegone, are well above average.

Designing a Science Operations structure for an Observatory is a little like designing a government, and a little like starting a business. From the outset we knew that our underlying "mission" was to facilitate the maximum science return from Spitzer. We didn't take a narrow view of that, but acknowledged that that meant understanding what was prudent in terms of operating the spacecraft as well as the science instruments. At the same time, we also recognized that the SSC had the role of advocating for making the maximum possible time available for science. I believe that our taking a active interest in understanding and acknowledging the importance of the health and safety of the spacecraft and the observatory as a whole encouraged the JPL Mission Operations Teams and the Observatory Engineering Team at Lockheed Martin Aerospace to take an active interest in maximizing the scientific return from Spitzer. It didn't hurt that these teams, also, have been outstanding performers.

Observatory Operations: Strategies, Processes, and Systems II, edited by Roger J. Brissenden, David R. Silva, Proc. of SPIE Vol. 7016, 70160C, (2008) - 0277-786X/08/\$18 $\cdot$ doi: 10.1117/12.789871 
So a combination of establishing checks and balances, combined with fostering a climate of understanding where each player has a role to play but understands that the opposing role is also important aids in efficient and cordial decisionmaking. Well, most of the time, anyway. In designing the operations processes and determining the roles and constitution of the teams, we kept this in mind and designed a system to facilitate gathering the relevant inputs and allowing appropriate weigh-in.

\section{ORGANIZATIONAL STRUCTURE}

SSC currently has the following (abstracted) Operations organizational structure:

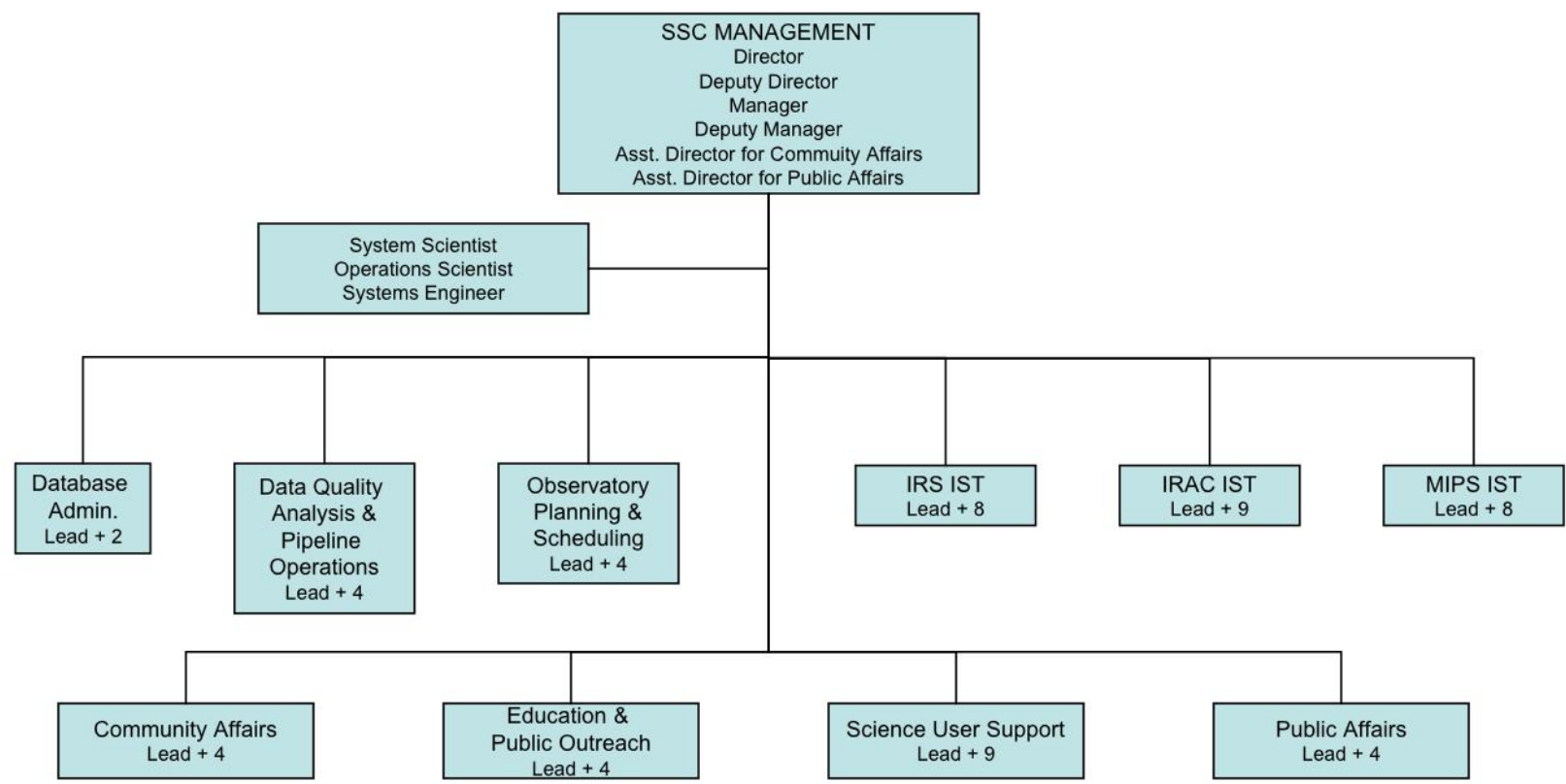

Figure 1 -- Schematic Current Operations Organization. Software development teams, administrative support, etc. have been omitted to simplify the chart and emphasize the operations teams.

\subsection{Current Operations Team Roles and Responsibilities}

\subsubsection{Database Administration}

Database Administration is responsible for maintenance of the Science Operations Database.

\subsubsection{Data Quality Analysis and Pipeline Operations}

Data Quality Analysis and Pipeline Operations combines the essentially separate Pipeline Operations and DQA teams into one management structure and covers keeping the pipelines running, performing a cursory analysis of science data quality and referring questionable data to the pertinent Instrument Support Teams and assigning a final quality status to the data. 


\subsubsection{Observatory Planning and Scheduling}

Observatory Planning and Scheduling (OPST) produces weekly observatory schedules for all normally scheduled activity including science, science instrument calibrations, downlinks and routine and periodic spacecraft calibrations. The end products are delivered to the Mission Sequence Team (MST) in the Mission Operations office at JPL at the command level for all but spacecraft activities that are not implemented as on-board blocks. The MST does the final safety checking and modeling of the sequences, and converts them into uplinkable form.

\subsubsection{Instrument Support}

The Instrument Support Teams (ISTs) are responsible for the health, safety and operations of the science instruments, including routine calibrations, special calibrations, trouble-shooting and supporting other operations teams as needed when instrument expertise is required. They retain support from the PI teams that built the instruments.

\subsubsection{Science User Support}

The Science User Support Team (SUST) is responsible for providing front-line support to users of Spitzer science data via workshops, a helpdesk, maintenance of web pages etc. and advocates for the observers in the context of observatory operations (e.g. scheduling).

\subsubsection{Community Support}

The Community Support Team is responsible for the programmatic aspects of supporting users of the science data including administering the Time Allocation process and funding.

\subsubsection{Public Affairs}

The Public Affairs Team is primarily responsible for interfacing with the press, the JPL press office and public events that utilize or showcase Spitzer results.

\subsubsection{Education and Public Outreach (EPO)}

The EPO Team is primarily responsible for educational K-12 programs. 


\subsection{Scheduling, an example of Checks and Balances}

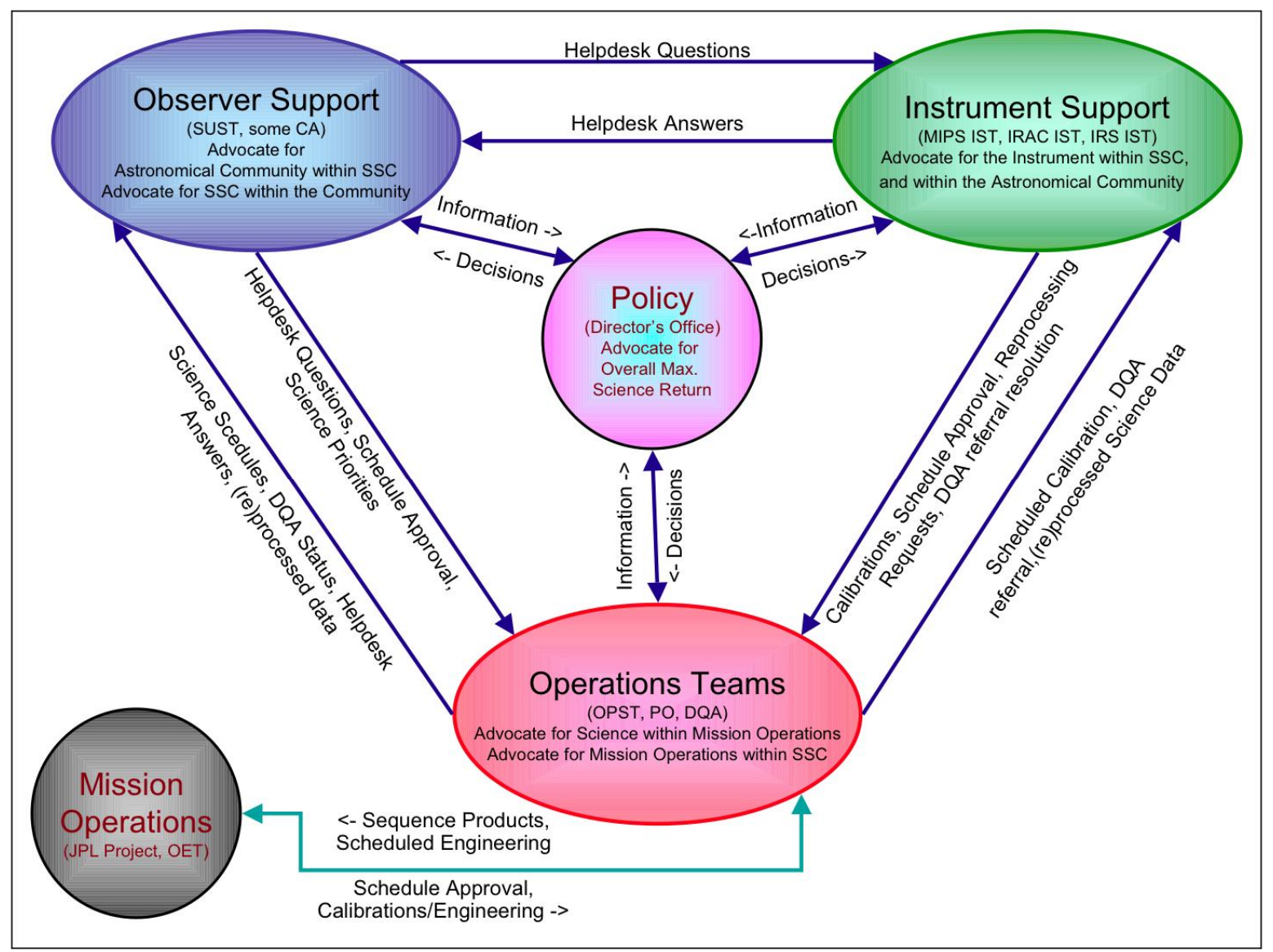

Figure 2 - Information and Decision Flow

It's useful to have different teams with different "constituencies" reviewing and approving operational decisions and products that significantly affect their primary base. For Spitzer (and most heavily lifetime constrained observatories) the most critical items are the routine science schedules and the handling of anomalies. Figure 2 illustrates how the flow of control works for Spitzer routine science schedules.

Science Schedules are prepared for Spitzer one week at a time. It takes about two weeks to bring a given schedule to the point of approval, which occurs two and a half weeks prior to uploading the command sequence. During that two and a half week period, the Mission Sequence Team at JPL takes the command sequence products delivered by OPST, adds commanding for special engineering events if necessary, models the commanding and packages the sequences into a final uplinkable form.

OPST sends out the schedule products for approval and then calls an approval meeting (nominally every Thursday morning). Typically, the schedule has been previously circulated at SSC for pre-review by the ISTs and SUST. The approval process is done during the meeting and the approvals are recorded along with any liens. Modifications may be made prior to delivery of the sequence products on Friday.

It turned out that the approval meeting was a very useful forum for discussion of operational issues affecting efficiency and science implementation, especially during the first year of operations. Although it was intended for a very specific 
purpose, it was the only weekly meeting that brought together the SSC Director, the Mission Manager, Instrument Support, Observer Support and OPST in a format conducive to detailed discussion.

The OPST does their best at balancing all the demands of the schedule and then submits it for approval. The SSC Director and the JPL Mission Manager are the two entities that have approval authority for the schedule, but the Science User Support Team, the Instrument Support Teams whose instruments are present in the schedule and the Observatory Engineering Team provide "supporting approvals" for the content of the schedule. The Instrument support teams are looking for correct scheduling of calibrations and maintenance activities (i.e. anneals) and correct handling of bright targets that might affect the quality of subsequent data. The Science User Support Team is looking for efficiency, correct handling of constraints, timely scheduling of programs and correct balance between observation types and priorities. The Observatory Engineering Team looks for correct scheduling of spacecraft and pointing system calibrations (usually providing their input via email.) The ISTs and SUST approvals are supporting the SSC Director's decision to approve or disapprove and the OETs approvals are supporting the Mission Manager's decision. Technically, the SSC Director approves the science and science instrument content of the schedule and the Mission Manager approves the engineering content and safety. In practice, people in both roles have been diligent and taken an interest in the overall integrity, safety and efficiency of the schedule.

\section{PLANS THAT FELL BY THE WAYSIDE}

\subsection{Changes to the Organization and Team Structure}

Early plans for the SSC Structure looked more like this:

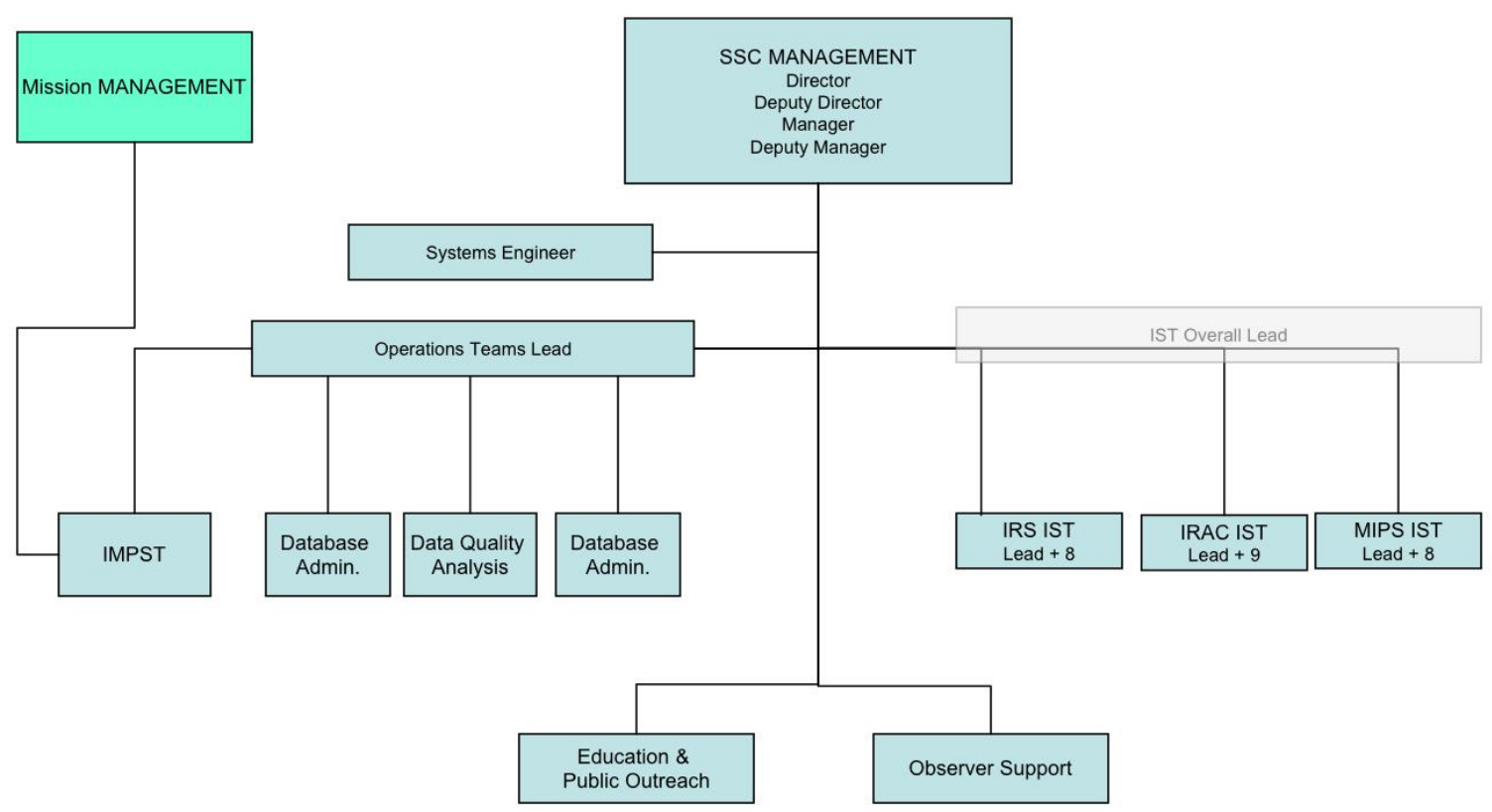

Figure 3 -- Prehistoric SSC Organization 
There was no Operations Scientist or System Scientist. There was an Overall Operations Teams Lead and DQA and PO were separate teams. There was one Observer support organization. There was briefly a combined Instrument Support Teams Lead, but only on scraps of paper. There was no Public Affairs group. There was no operational Database Administration Team. But perhaps most notably, there was an Integrated Mission Planning and Sequencing Team combining JPL and SSC staff members.

\subsubsection{Instrument Support Team Overall Lead Fails to Fly}

The idea of a single lead overseeing the schedules and work of all three science instruments proved unpalatable early on and the idea was discarded. The ISTs felt they needed autonomy to properly support their specific instrument. The role of System Scientist evolved to fill the niche of providing an individual charged with maintaining a technical/scientific overview of science center operations (including the science instruments, observatory pointing performance, etc.) without having supervisory status for the ISTs.

\subsubsection{The Integrated Mission Planning and Scheduling Team (IMPST) Disintegrates}

The IMPST concept also fell apart prior to launch. It is the impression of this author that this is a repeating theme in space-based observatories. While having one team take the whole of spacecraft scheduling and sequencing from blank slate to uplinkable product is demonstrably more efficient, it requires giving up, or at least gives the impression of giving up, a certain amount of control. If a joint team is lead by Science Operations, Mission Operations tends to feel it is giving up control over the safety and engineering aspects of the sequence. If a joint team is led by Mission Operations, Science tends to feel it is giving up control over the scheduling of the science. A number of different solutions to this were proposed, but none of them proved acceptable to both parties and current division of responsibilities was the result. For Spitzer, the division of responsibilities actually gives a great deal of responsibility to the OPST, an SSC Team, and allowing that team to place engineering activities in the timeline integrated with the science is one reason Spitzer has been able to achieve the impressive on-orbit efficiencies it has. The fact that science activities are handled based on their actual command expansions from the get-go (time estimates provided to proposers are based on commanding) and the project uses a common pointing model for both science and mission operations is another. Having a unified team would have further facilitated these things, but we were able to coordinate them anyway. The primary down side to the OPSTMST structure is that the timeline from initial start of scheduling to uplink is 1-2 weeks longer than could be done with an integrated team (some of this additional lead time was reduced once operations became smoother and trust was better established) and it takes 2-4 more staff members to provide sufficient coverage.

This author still believes that the integrated concept is the best way to handle scheduling and sequencing for an observatory and notes that HST operations have evolved towards this arrangement. The IMPST paradigm is being reevaluated for the Spitzer Warm Mission.

\subsubsection{Database Administration becomes an Operations Team}

Initially, the database administration function for the Science Operations Database was performed within the development team working on the database. However, the SODB is central to science operations and activities such as down time and tuning had a strong impact on operations. Prior to launch this became apparent, and the DBA team became an operations team managed under the Operations Teams umbrella.

\subsubsection{The Operations Teams Lead becomes Operations Scientist and the teams become independent}

The Operations Teams Lead was initially the Operations Planner and that role segued into providing supervisory oversight to all four teams (OPST, PO, DQA and DBA) for the early part of the mission. The same individual also acted as lead for the OPST. As science operations began to settle into more of a stable routine, the team leads began to function more and more independently. In addition, the Ops Teams Lead was spending much time interfacing with Mission Operations. It made sense to transfer line management of the teams to the team leads themselves, to appoint a lead for OPST and to transition the role of Ops Teams Lead to Operations Scientist.. At this time PO and SDQA were combined under the same Lead. This latter decision was driven partly by the specific wishes and talents of the staff members involved.

The Operations Scientist has a primary charge of overseeing the "big picture" of science operation and interfacing with Mission Operations. The Operations Scientist is the SSC point person for anomaly response. 


\subsubsection{Assistant Directors for Community Affairs and Public Affairs are Named}

Early in the mission, the director appointed individuals to concentrate on these two areas. Initially they had no dedicated staff, but worked with the Observer Support and EPO staffs.

\subsubsection{Observer Support becomes Science User Support and Community Affairs}

These teams split after a period of operations with both sets of responsibilities in a single team. A combination of the different and somewhat disjoint schedule demands placed by ongoing support and the TAC process and the specific talents and working styles of the staff members involved lead to formally splitting out the responsibilities into two teams with separate leadership. This has worked very well for Spitzer. The Assistant Director for Community affairs became lead for the community affairs team.

\subsubsection{Public Affairs springs forth from EPO}

Originally EPO covered both K-12 and general educational activities as well as some coverage of press activities and other publicity activities. Interfacing with the press process turned out to need dedicated time and effort, and this team was split out and staffed up shortly after launch. The Assistant director for Public affairs became lead for the PA team.

\subsection{Changes to operational strategies and Plans}

In this section is a selection of operational changes made during early planning and during operations.

\subsubsection{Reduction in Scope of Event Driven Execution}

Early plans had much of the scheduling "smarts" on-board the spacecraft. The Science Center would select a list of viable observations to schedule, plus some extra for efficiency, and that would be loaded to the spacecraft, which would figure out what to execute. SSC was concerned about predictability of scheduling of science priorities and wanted more control over the ordering and content. By the time we launched, the degree of remaining onboard autonomy had been reduced to a slew complete and a pointing stable indicator.

From that point, we have eliminated use of even the stability indicator, as we could be more efficient with a fixed timeout. However, we have added functionality to prevent filling the mass memory.

\subsubsection{Reduction in scope of Automated Scheduling}

Early Plans also had the scheduling software doing most of the actual scheduling, with a human in the loop only to double-check and tweak the results. In practice, more efficient schedules could be made with the human scheduler manually setting up a "skeleton" of required activities, allowing the automated system to fill in the schedule and then hand-tweaking the final result. The decision support aspects of the scheduling software are very important in this scenario.

\subsubsection{Allowing the Telescope Temperature to Float; Pulsing the make-up heater}

Prior to launch it had been recognized that since only $160 \mu \mathrm{m}$ observations require the telescope to be at it's coldest (5.5K), and since the telescope is cooled by blowing off cryogen using a heater to dissipate additional power, adding to that put in by the science instrument itself, in the cryostat, lifetime could be optimized by not turning on that heater (known as the make-up heater) and allowing the telescope to warm up when we weren't running the long-wavelength science instrument (MIPS). The lifetime increase could be optimized by scheduling the other two instruments in order of increasing power dissipation. Somewhat later it was determined that the most efficient way (in terms of amount of cryogen used) to cool the telescope back down for MIPS observations was to put in a short duration high heater level and then adjusting the heater to the maintenance value for the cold telescope. These activities are scheduled by OPST using a tool provided by the Observatory engineering Team

\subsubsection{Further increasing lifetime - Warm and Cold MIPS}

After Spitzer had been operating a while, it became clear that only a subset of MIPS observations, less than half, were making use of $160 \mu \mathrm{m}$ data. Additional lifetime could be eked out by separating MIPS campaigns into cold and "warm" $(\sim 7 \mathrm{~K})$.

\subsubsection{Eliminating Pad - The Slave Halt}

In order to avoid invoking fault protection should a science activity run longer onboard than predicted on the ground, due to imperfect modeling of the slew complete and pointing ready indicators, it was initially necessary to add "pad" or 
scheduled dead time to the schedule/sequences. In order to allow us to make best statistical use of these indicators and ramp back on the padding, we built sequence constructs that would clean up themselves without generating a fault condition. This was another contributor to high efficiency.

\subsubsection{Keeping the Mass Memory from filling}

Perhaps the biggest paradigm shift we encountered during operations was the need to actively manage data storage. We had believed that Spitzer would benignly over-write older data that had not been acknowledged by the ground should we collect more data than predicted. However, fairly late during operations planning, it was realized that over-writing unacknowledged data could have unpredictable consequences including significant fault protection activity. In addition, in practice it was not possible to acknowledge the data that had been downlinked during the same session in which it was downlinked, so the spacecraft always came in to a downlink carrying twice the average amount of data collected between downlinks. Should a downlink be missed, it would then be carrying three times that amount. As we became more efficient, data volume, especially for MIPS, frequently was at a level where efficiency would need to be compromised or very careful balancing of low and high volume observations would need to be done in order to avoid risking filling up the memory if a downlink pass was missed. To make this particular problem even more interesting, the amount by which a given set of data would compress was not predictable because it depended on the statistical characteristics of that unique observation! And as Spitzer gets farther away from Earth, the link margins for downlink get tighter, and communication relies on the largest DSN antennas, so the risk of missing a downlink becomes more significant.

Although we could not do anything to completely eliminate the risk of filling the memory, we were able to find a way to cause the onboard sequence to stop collecting science data if a given observation was predicted to collect more data than the remaining free space available. If a subsequent observation would collect less data, it could proceed to execute. This limited the risk from filling the mass storage to missing observations rather than recovering the spacecraft from an anomaly.

\subsubsection{Dealing with bright objects}

Bright objects were the other initially unanticipated wrinkle in scheduling. It was initially believed that no lingering effects would remain after observing a saturating source for any of the science instruments. In practice, however, latent images did occur in some cases and were significant enough to interfere with very sensitive observations of faint targets. Initially, observations of bright objects were manually identified by the ISTs at the time of schedule review and were moved as needed. Later more tools were built into the scheduling software to help identify these observations earlier in the process so the ISTs needed only to double check them at schedule approval time.

\section{IN CONCLUSION: THE GOOD, THE BAD AND THE UGLY}

There is much that has been good about Spitzer operations. The most easily measured attribute attesting to this is the onorbit efficiencies in excess of $90 \%$. Spitzer is generally well thought of in the community and other observatories have adopted some of our processes and tools.

One of the less easily observed good things has been the excellent working relationship between the SSC and the OET and mission operations. This good working relationship led to a truly collaborative effort to maximize efficiency without compromising safety. It has enabled us to work well together both during routine operations and when responding to anomalies.

As for the bad and the ugly, well, the surprises did present us with a headache, and we are constantly drawing on our good working relationship to squeak us past filling mass memory and skipping science, but the good remains the dominant force.

Despite the changes made along the way, the planning that was done before launch was key in setting the stage for the very successful operations we have enjoyed. Working together and hashing through problems together created teams that understood how to work together. Working through the details of plans enabled us to understand the details of how things really work, and that laid the groundwork for future improvements.

Dwight D. Eisenhower had it right when he uttered this statement, "When it comes to battle, I find that plans are useless but planning is essential." 